\title{
FACTORES DETERMINANTES DEL DESEMPEÑO EMPRESARIAL EN LIMA METROPOLITANA DURANTE LA PANDEMIA DEL COVID-19
}

\author{
DETERMINANTS OF BUSINESS PERFORMANCE IN METROPOLITAN LIMA DURING \\ THE COVID-19 PANDEMIC
}

JoSHEP IVAn RAmos ReYES

Moore Perú

Lima, Perú

ORCID: https://orcid.org/0000-0002-6571-0447

Correo electrónico: jramos@moore-peru.pe

\section{RESUMEN}

Objetivo: Identificar los factores determinantes que explican el bajo desempeño empresarial en Lima Metropolitana durante la pandemia del COVID-19. Método: Se utilizó el análisis factorial para agrupar los problemas que enfrentaron las empresas de Lima Metropolitana durante la pandemia del COVID-19, y también para identificar los principales factores asociados a estos problemas. Luego se estimaron los efectos que tuvieron los factores sobre el desempeño empresarial, entendido como el índice de ventas de las empresas. Resultados: Los factores asociados a los problemas de liquidez y operatividad tuvieron un efecto negativo y estadísticamente significativo sobre el índice de ventas de las empresas. Sin embargo, el factor asociado a los problemas de liquidez fue determinante para explicar el comportamiento del índice de ventas en las empresas de Lima Metropolitana durante la pandemia del COVID-19. Conclusión: Los problemas asociados al acceso a financiamiento y falta de liquidez de las empresas son los que explican en mayor medida el bajo desempeño empresarial en Lima Metropolitana durante la pandemia del COVID-19.

Palabras clave: Financiamiento; empresas; problemas; COVID-19.

\begin{abstract}
Objective: Identify the determining factors that explain the poor business performance in Metropolitan Lima during the COVID-19 pandemic. Method: Factor analysis was used to group the problems that Metropolitan Lima companies faced during the COVID-19 pandemic, and to identify the main factors associated with these problems. Subsequently, the effects that the factors had on business performance were estimated, which are understood as the companies' sales index. Results: The factors associated with liquidity and operational problems had a negative and statistically significant effect on the companies' sales index. However, the factor associated with liquidity problems was decisive in explaining the behavior of the sales index in companies in Metropolitan Lima during the COVID-19 pandemic. Conclusion: The problems associated with access to financing and lack of liquidity of companies are the ones that explain to a greater extent the poor business performance in Metropolitan Lima during the COVID-19 pandemic.
\end{abstract}

Keywords: Financing; companies; problems; COVID-19.

(C) Los autores. Este artículo es publicado por la revista Quipukamayoc de la Facultad de Ciencias Contables, Universidad Nacional Mayor de San Marcos. Este es un artículo de acceso abierto, distribuido bajo los términos de la licencia Creative Commons Atribución 4.0 Internacional (CC BY 4.0) [https://creativecommons.org/licenses by/4.0/deed.es] que permite el uso, distribución y reproducción en cualquier medio, siempre que la obra original sea debidamente citada de su fuente original. 


\section{INTRODUCCIÓN}

La empresa es una unidad técnica o unidad de organización dedicada a la realización de actividades productivas y como tal transforma un conjunto específico de factores o insumos para producir bienes o servicios que se ofrecen en el mercado. Como afirma Coase (1994), "la empresa, en la teoría económica moderna, es una organización que transforma factores de producción en producción" (pp. 12-13). Estos factores son: tierra (suelo, subsuelo, agua, pluviosidad, clima), trabajo, capital, capacidad empresarial y capacidad tecnológica.

Además, las empresas deben adoptar una forma societaria con el objeto de operar en el mercado; es decir, pueden constituirse como sociedades anónimas, sean estas abiertas o cerradas, empresarios individuales de responsabilidad limitada, sociedades comerciales de responsabilidad limitada, etc. Al respecto, Brealey, Myers y Allen (2010) señalan que existen diferencias organizacionales dependiendo del tamaño de la empresa. Así, las medianas y grandes empresas funcionan como corporaciones, mientras que las micro y pequeñas empresas cuentan, principalmente, con un propietario individual.

En particular, las pequeñas empresas - en todas sus formas - se caracterizan por su reducida escala de explotaciones e inversiones o la escasa importancia económica de sus actividades; asimismo, cuentan con menor capital social y menores posibilidades de acceder a créditos en condiciones más favorables, como son los casos de las medianas o grandes empresas.

En las pequeñas empresas, frecuentemente de carácter familiar, el empresario es a la vez propietario de la empresa y es quien la dirige. La doble condición de propietario y administrador implica que asume un riesgo patrimonial ya que su objetivo es hacer rentable el dinero invertido, que al menos parcialmente es suyo. (Mochón, 2009, p. 117)

Por su parte, Brealey et al. (2010) argumentan que, para hacer negocios, las corporaciones necesitan de activos reales, los cuales pueden ser tangibles o intangibles. Entre los primeros, se presenta la maquinaria utilizada, las instalaciones y oficinas; mientras que en los activos reales intangibles, se encuentran la capacidad de la fuerza laboral, la capacidad de gestión, el valor de la marca y la especialización de los trabajadores. Para acceder a estos activos reales, es necesario que las empresas puedan hacer uso de diversos mecanismos y de un instrumento para permitir su financiamiento, el cual puede ser con capital propio o a través de los mercados financieros.
Por otro lado, Regente (2020) analiza el comportamiento de las microempresas tomando en cuenta sus características. Así, estimó un modelo econométrico de regresión lineal múltiple, en donde toma como endógena la variable variación anual de las ventas de la empresa y, como exógena, un vector de variables características de la empresa y del microempresario que conduce la actividad, tales como: acceso a crédito, capacidad productiva, la educación y capacitación del microempresario, la fuerza laboral en la microempresa, la educación y capacitación de los trabajadores. Como principal resultado, el autor halla efectos positivos y estadísticamente significativos en las variables de acceso al crédito, la capacidad productiva, la educación del microempresario y la fuerza laboral en la empresa. De esta manera, concluye que el crecimiento de las ventas en las microempresas textiles de Arequipa es explicado por el número de trabajadores de la empresa, la capacidad productiva, el nivel educativo del microempresario y el acceso al crédito. Por ende, se puede observar la importancia que tienen la capacidad productiva y el acceso a los mecanismos de financiamiento para el crecimiento empresarial.

Sin embargo, considerando que las empresas se desarrollan en un entorno que también responde a factores externos, Periche-Delgado, Ramos-Farroñan y Chamolí-Falcón (2020) encontraron que las empresas en el Perú enfrentaron diversas restricciones durante la pandemia del COVID-19 y ello resultó en el incremento del nivel de morosidad. Este aumento también se traduce en futuras limitaciones de acceso a mecanismos de financiamiento por parte de las empresas a través del sistema financiero formal en el Perú.

En la misma línea, Quilia (2020) realizó un estudio que buscó analizar los desafíos empresariales de las mype durante la pandemia del COVID-19 el año 2020 en el Perú. El autor encontró que la pandemia del COVID-19 trajo consigo desafíos a la gestión empresarial, especialmente, en torno a la preparación ante un evento de magnitud global que conlleva la existencia de riesgos covariados para los cuales no se encontraban preparados, en especial, las mype. Esta situación afectó más su condición de vulnerabilidad. Por otro lado, existieron también desafíos con relación al financiamiento, lo cual afectó la liquidez de las empresas a causa de la política de paralización de actividades designadas, impuesta por el Gobierno, con el propósito de proteger a la población. Esta situación trajo consigo el incumplimiento en el pago de deudas a proveedores, lo cual incrementó el reto de la gestión empresarial bajo estas condiciones. De esta manera, se observa que los factores más destacados para las empresas no solo se relacionan con el financiamiento, sino con la gestión empresarial o la operatividad de la empresa. 
De igual forma, aproximadamente 600 mil empresas cerraron durante el año 2020 a causa de la pandemia del COVID-19, de las cuales casi la totalidad se encuentran en el segmento de las microempresas (Instituto Peruano de Economía, 2021). Esta situación incrementó en gran medida el riesgo de crédito asociado a todas las empresas en el Perú, afectando en mayor proporción a las microempresas.

Asimismo, se observa que este escenario no es exclusivo del Perú, pues Franco, Gómez y Serrano (2019) hallaron para Ecuador que, a pesar de que las pymes conforman una parte importante del tejido empresarial y son un factor relevante para el crecimiento económico del mismo, no acceden en gran medida al financiamiento a través del sistema financiero. Así, obtienen como principales resultados que la probabilidad de acceder a un crédito se ve afectada por la escala de su producción, de manera que, una empresa mediana tiene más probabilidades de acceder a un crédito en comparación con una empresa pequeña.

Por otro lado, Achim, Safta, Văidean, Mureșan y Borlea (2021) analizaron la relación de diversos factores sobre el nivel de desempeño comercial de las empresas en Rumania durante la pandemia del COVID-19. Sus resultados muestran que el financiamiento de capital, la liquidez, adecuada gestión y un mayor tamaño de la empresa consolidan el desempeño de las empresas. Además, los autores consideran que los Gobiernos deben saber cómo responden las empresas a la pandemia, así como identificar los sectores más vulnerables a los efectos de la crisis y las principales decisiones de gestión financiera que deben ser adoptadas por las empresas en tiempos de crisis.

En ese sentido, se muestra que, en particular, las grandes empresas tienen menores problemas asociados con el acceso a mecanismos de financiamiento, en comparación con empresas pequeñas, en condiciones normales diferentes a la acaecida producto de la pandemia del COVID-19. En ese sentido, cada empresa enfrenta un distinto set de restricciones, derivado de sus actividades económicas, escala de producción, capacidad de gestión y operatividad, entre otros; en consecuencia, hará frente de distinta forma a los eventos externos que se presenten, como las restricciones acarreadas a causa de la pandemia del COVID-19.

Por su parte, Ullah (2021) encuentra que las empresas con restricciones crediticias eran más propensas a que utilicen crédito comercial, retrasen los pagos a proveedores o empleados y dependan de subvenciones del Gobierno para hacer frente a los problemas de liquidez y flujo de caja relacionados con la pandemia. Para el autor, había poca evidencia de que las empresas con racionamiento de crédito tuvieran más probabilidades de obtener capital social durante esta crisis económica.
En este sentido, el presente estudio busca responder la siguiente interrogante: ¿Cuáles son los factores determinantes que explican el desempeño empresarial en Lima Metropolitana durante la pandemia del COVID-19? Para tal efecto, se busca corroborar la siguiente hipótesis de investigación: "Los principales factores que explican el desempeño empresarial en Lima Metropolitana durante la pandemia del COVID-19 son aquellos referidos a las limitaciones en la producción y al financiamiento".

\section{MATERIAL Y MÉTODOS}

El enfoque fue cuantitativo debido a que se usaron mediciones para contrastar las hipótesis y se aplicó un diseño no experimental debido a que las variables de estudio no fueron manipuladas. El alcance del estudio fue descriptivo y explicativo. Fue descriptivo en la medida que buscó analizar las características de las empresas en Lima y Callao en términos de tamaño de empresa y actividad económica desempeñada; además, observó cuáles fueron los problemas que enfrentaron las empresas en Lima y Callao a causa de la pandemia del COVID-19 durante el segundo trimestre de 2020. Fue explicativo debido a que se indagó sobre los factores asociados a la pandemia del COVID-19 que afectaron el normal desempeño de las empresas durante el segundo trimestre de 2020. Para tal fin, la población de estudio estuvo conformada por las empresas de Lima Metropolitana (Lima y Callao) y la unidad de análisis fue la empresa.

Se utilizó la Encuesta de opinión sobre el impacto del COVID-19 en las empresas (EOIC19) (Instituto Nacional de Estadística e Informática, 2020). La EOIC19 recaba información acerca de los problemas, expectativas y percepciones que tuvieron más de 240 mil empresas en Lima Metropolitana. En particular, el estudio se centró en los problemas que afrontaron 929 empresas por causa del COVID-19 en el segundo trimestre 2020 (abril-mayo-junio). Cabe resaltar que las variables que identifican los problemas son todas de orden dicotómico.

Asimismo, se construyó un índice de ventas, el cual es una variable continua que representa el desempeño empresarial durante este periodo, a partir de la información de dos variables referidas a: $i$ El nivel de ventas de la empresa en el segundo trimestre 2020 (abril-mayo-junio) con respecto al segundo trimestre de 2019 (abril-mayo-junio) aumentó, se mantuvo o disminuyó? y ¿en qué porcentaje? En donde el índice de ventas toma un valor de 100 si no hubo cambios en las ventas, mayor a 100 si creció y menor a 100 si disminuyó.

A partir de la información y variables referidas a los problemas que enfrentaron las empresas, se procedió a realizar el análisis que tuvo un componente descriptivo y 
otro inferencial. El análisis descriptivo buscó mostrar las principales características de las empresas en Lima Metropolitana durante la pandemia del COVID-19, tales como la composición del grupo de empresas tanto por tamaño como por sector económico, así como la incidencia de los problemas señalados por las empresas en esta área. Asimismo, se indagó sobre el comportamiento de las ventas que tuvieron las empresas a partir del índice de ventas antes descrito, y la condición de operatividad en la que se encontraban estas empresas en el mismo periodo.

Luego de ello, se efectuó el análisis inferencial que buscó hallar cuáles fueron los factores que incidieron sobre el desempeño empresarial, aproximado por el índice de ventas, durante la pandemia del COVID-19. Este análisis inferencial tuvo dos partes: la primera referida al análisis factorial con el propósito de reducir las dimensiones y hallar comunalidades entre los distintos problemas que enfrentaron las empresas en Lima Metropolitana durante la pandemia; y la segunda utilizó una regresión lineal para identificar cuál de los factores previamente hallados sirven para explicar mejor el desempeño de las empresas durante el mismo periodo.

Siendo así, se realizó un ajuste al método tradicional del análisis factorial, debido a que al ser todas las variables dicotómicas - referidas a los problemas que afrontaron las empresas - hacen inapropiado el uso de la correlación de Pearson. De esta manera, se usó la correlación policórica, indicada para medir las correlaciones entre variables categóricas para hallar la matriz de correlación que sirvió de insumo para el análisis factorial. Además, para comprobar la adecuación del uso del análisis factorial, se utilizaron dos estadísticos: la medida de adecuación muestral de Kaiser-Meyer-Olkin (KMO) y la prueba de esfericidad de Barlett. Por ello, si los datos tienen estructura factorial, el estadístico KMO tendrá un valor más cercano a 1 y tenderá a 0 en caso contrario. Es necesario recalcar que, para usar el análisis factorial, el valor mínimo aceptable del estadístico KMO es 0.6. Por otro lado, la prueba de esfericidad de Bartlett tiene por hipótesis nula que: "No hay correlaciones entre las variables". Asimismo, se evalúa de acuerdo con el p-valor de la prueba Chi-cuadrado: si es menor a 0,05 , se rechaza la hipótesis nula y se corrobora que existen correlaciones entre las variables.

Seguidamente, mediante el análisis de factores, se eligió el número óptimo de factores sobre la base de aquellos autovalores que superen el valor de 1 que, en conjunto, sirvan para explicar la mayor parte de la varianza del grupo de variables. Luego, se obtuvo la carga de factores, la cual fue rotada para optimizar la relación entre los factores hallados y las variables, procediendo así a su identificación con las variables referidas a los problemas que enfrentaron las empresas en Lima Metropolitana durante la pandemia del
COVID-19. A partir de ello, se predijo el valor de los factores hallados previamente para cada una de las empresas en la muestra estudiada.

Finalmente, se estimó un modelo de regresión lineal mediante mínimos cuadrados ordinarios (MCO) - siguiendo a Cameron y Trivedi (2009) - con el propósito de hallar cuáles fueron los factores más importantes que afectaron el desempeño de las empresas en Lima Metropolitana durante la pandemia del COVID-19. Así, el modelo de regresión consideró, como variable endógena continua, el desempeño empresarial medido como el índice de ventas de las empresas descrito en párrafos anteriores $\mathrm{y}$, como vector de variables exógenas, los factores hallados a partir del análisis factorial. De esta manera, se estimó la siguiente función:

$$
\mathrm{Y}=\alpha+\beta^{*} \text { Factor }_{\mathrm{i}}
$$

En donde "Y" es el índice de ventas; "Factor" son los factores hallados previamente $y$ " $\beta$ " es el coeficiente asociado al i-ésimo factor. Siendo así, el coeficiente " $\beta$ ” indicó la existencia, sentido y magnitud del efecto que cada factor tenía sobre el índice de ventas de las empresas y permitió identificar cuáles eran los factores determinantes y estadísticamente significativos que explican el desempeño empresarial en Lima Metropolitana durante la pandemia del COVID-19. Se observó la significancia estadística de los coeficientes tomando en cuenta que el p-valor de los “ $\beta$ " sea menor a 0,05. Además, la bondad de ajuste del mo-

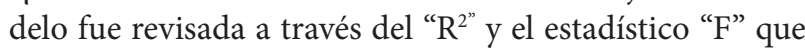
indica la significancia conjunta del modelo.

\section{RESULTADOS}

A partir de la EOIC19, se observa que el tejido empresarial de Lima Metropolitana está compuesto, en su mayoría, por micro y pequeñas empresas, las cuales representan más del 95\% del total, mientras las grandes empresas constituyen menos del 3\%. Ello refleja el grado de vulnerabilidad que enfrenta el grupo de empresas en Lima Metropolitana. Además, la EOIC19 muestra que la mayor parte de las empresas de dicha área se dedica al comercio (46\%), seguido de las actividades inmobiliarias (16\%), transporte, almacenamiento y comunicaciones $(10,8 \%)$ e industria manufacturera (10,8\%). Asimismo, las empresas dedicadas al sector de hotelería y restaurantes representan el 6\% del total de empresas. De esta manera, se resalta que las actividades del sector terciario predominan en Lima Metropolitana.

Por otro lado, en la Tabla 1, se observa que más del 75\% de las empresas en Lima Metropolitana vieron afectadas su normal funcionamiento. Así, $24 \%$ de las empresas resultaron inoperativas durante la pandemia del COVID-19 y 
$53 \%$ señalaron que se encontraban parcialmente operativas. Solo $23 \%$ de las empresas ubicadas en Lima Metropolitana señalaron que estaban en condición operativa. Esta situación guarda relación con lo observado previamente en lo referido a la vulnerabilidad del tejido empresarial en Lima Metropolitana vinculada al tamaño de empresas y sectores económicos predominantes.

Tabla 1

Empresas por estado de operatividad

\begin{tabular}{lcc}
\hline Estado de Operatividad & $\mathbf{N}$ & $\mathbf{\%}$ \\
\hline Operativa & 54,561 & $22,72 \%$ \\
Parcialmente operativa & 126,887 & $52,83 \%$ \\
Inoperativa & 58,745 & $24,46 \%$ \\
Total & $\mathbf{2 4 0 , 1 9 4}$ & \\
\hline
\end{tabular}

Fuente: Elaboración propia a partir de datos de la EOIC19 (Instituto Nacional de Estadística e Informática, 2020)

En ese contexto, más del 70\% de las empresas vieron afectado su nivel de ventas con relación al año 2019. En la Tabla 2 , se muestra que $52 \%$ de las empresas señalaron haber disminuido sus ventas y $21 \%$ no realizaron ventas durante el segundo trimestre del año 2020. Por otro lado, solo $10 \%$ mantuvo su nivel de ventas en comparación con el obtenido en el año 2019, e incluso un 5\% incrementó sus ventas en contraste con el mismo periodo.

Tabla 2

Nivel de ventas durante la pandemia del COVID-19

\begin{tabular}{lcc}
\hline Nivel de ventas de la empresa & $\mathbf{N}$ & $\mathbf{\%}$ \\
\hline Disminuyó & 124,020 & $51,63 \%$ \\
Aumentó & 13,039 & $5,43 \%$ \\
Se mantuvo & 24,877 & $10,36 \%$ \\
No realizó ventas & 49,506 & $20,61 \%$ \\
Missing & 28,752 & $11,97 \%$ \\
Total & $\mathbf{2 4 0 , 1 9 4}$ & \\
\hline
\end{tabular}

Fuente: Elaboración propia a partir de datos de la EOIC19 (Instituto Nacional de Estadística e Informática, 2020)

El índice de ventas de las empresas en Lima Metropolitana muestra que, en promedio, las ventas de las empresas fueron afectadas negativamente en más del 50\% en comparación con el año 2019. En ese sentido, se observa que el índice de ventas fue en promedio $54,6 \%$ y tuvo una mediana de $40 \%$. Eso muestra que, a pesar de que las empresas estuvieron operativas y realizaron ventas, más del $50 \%$ de estas declaró que tuvo caídas de más de $60 \%$ de sus ventas en comparación con el periodo anterior.

Este resultado condice con lo expresado en la Tabla 3, en donde se muestran los principales problemas que enfrentaron las empresas de Lima Metropolitana durante la pandemia del COVID-19. Se observa que $70 \%$ de las empresas declararon que el principal problema durante el segundo trimestre del año 2020 fue causado por la caída de la demanda de productos y servicios. Más del $40 \%$ de las empresas enfrentaron problemas como los altos costos para implementar los planes de seguridad (51\%), requeridos para el funcionamiento de las empresas y el desarrollo de sus actividades; la paralización de la producción a causa de la pandemia (48\%), producto de las restricciones de tránsito y distanciamiento social dictaminado por el Gobierno; el retraso de pago y cobro de facturas; así como la dificultad para cobrar a sus clientes. Además, otros problemas que tuvieron más del $30 \%$ de las empresas estuvieron asociados a las limitaciones para acceder al financiamiento y la dificultad de pagar los préstamos en el sistema financiero. Ello guarda relación con las limitaciones que tuvieron las empresas para realizar el normal funcionamiento de sus operaciones y, por ende, afectaron sus ingresos y posibilidades para acceder a mercados de insumos como el de crédito.

El análisis factorial utilizó la correlación policórica, tal como se describió en la sección de material y métodos. Los resultados de la aplicación de la correlación policórica a las variables referidas a los problemas enfrentados por las empresas en Lima Metropolitana durante la pandemia se muestran en la Tabla 4, bajo la forma de una matriz de correlaciones, en donde el nombre de las variables está expresado de acuerdo con los valores de la Tabla 3. Los resultados muestran que existen correlaciones entre las variables referidas a los problemas enfrentados durante la pandemia del COVID-19 y estas son positivas y moderadas en casi su totalidad.

La Tabla 5 señala el resultado de las pruebas estadísticas del test de esfericidad de Barlett y la medida de adecuación muestral de Kaiser-Meyer-Olkin. Así, p-valor del test de Barlett corrobora la existencia de correlación entre las variables de estudio y la idoneidad del uso del análisis factorial. Además, el estadístico $\mathrm{KMO}=0,842$, mayor al valor mínimo aceptado de 0,6 , por lo cual, la adecuación muestral es meritoria y resulta pertinente el uso del análisis factorial. Ello demuestra que es apropiado continuar con la aplicación del análisis factorial.

Luego, para estimar el número óptimo de factores, se analizaron los autovalores. De esta manera, en la Tabla 6 se muestra que solo tres factores tienen autovalores mayores a 1 y estos 3 en su conjunto sirven para explicar el 91,26\% de la varianza. Por tal motivo, el análisis señala que el número óptimo de factores es 3.

A partir de ello, se analizaron las cargas factoriales para observar las relaciones que existen entre las variables analizadas y los factores encontrados. Luego, se aplicó 
Joshep Ivan Ramos Reyes

Tabla 3

Problemas enfrentados durante la pandemia del COVID-19

\begin{tabular}{clc}
\hline Variable & \multicolumn{1}{c}{ Problemas enfrentados durante la pandemia del COVID-19 } & $\%$ \\
\hline V1 & Disminución de la demanda de sus productos o servicios & $69,92 \%$ \\
V11 & Altos costos para implementar planes de seguridad (personal y/o clientes) & $51,18 \%$ \\
V2 & Paralización de la producción a causa de la pandemia & $48,16 \%$ \\
V8 & Retraso en el cobro de facturas & $46,05 \%$ \\
V7 & Retraso en el pago de facturas & $42,04 \%$ \\
V18 & Dificultad para cobrar a sus clientes & $40,26 \%$ \\
V14 & Limitaciones para acceder a fuentes de financiamiento & $39,73 \%$ \\
V10 & Pérdida de capital de trabajo & $39,41 \%$ \\
V13 & Falta de liquidez para la compra de insumos o materias primas & $38,42 \%$ \\
V17 & Falta de liquidez para pagar a proveedores & $35,75 \%$ \\
V15 & Falta de liquidez para pago de remuneraciones de personal & $34,33 \%$ \\
V16 & Dificultad para pagar préstamos del sistema financiero & $28,21 \%$ \\
V12 & Difícil accesibilidad a créditos de sus proveedores & $26,05 \%$ \\
V4 & Desabastecimiento de materias primas e insumos del mercado nacional & $23,62 \%$ \\
V5 & Desabastecimiento de materias primas e insumos del extranjero & $18,76 \%$ \\
V9 & Enfermedad de trabajadores por el coVID-19 & $16,26 \%$ \\
V6 & Disminución de la inversión en maquinaria y equipos & $13,33 \%$ \\
V3 & Dificultad en la exportación de sus productos & $8,17 \%$ \\
\hline
\end{tabular}

Fuente: Elaboración propia a partir de datos de la EOIC19 (Instituto Nacional de Estadística e Informática, 2020)

Tabla 4

Matriz de correlaciones policóricas

\begin{tabular}{|c|c|c|c|c|c|c|c|c|c|c|c|c|c|c|c|c|c|c|}
\hline & V1 & V2 & V3 & V4 & V5 & V6 & V7 & V8 & V9 & V10 & V11 & V12 & V13 & V14 & V15 & V16 & V17 & V18 \\
\hline V1 & 1.00 & & & & & & & & & & & & & & & & & \\
\hline V2 & 0.13 & 1.00 & & & & & & & & & & & & & & & & \\
\hline V3 & 0.08 & 0.30 & 1.00 & & & & & & & & & & & & & & & \\
\hline V4 & 0.20 & 0.47 & 0.48 & 1.00 & & & & & & & & & & & & & & \\
\hline V5 & 0.09 & 0.27 & 0.51 & 0.68 & 1.00 & & & & & & & & & & & & & \\
\hline V6 & 0.41 & 0.33 & 0.33 & 0.42 & 0.51 & 1.00 & & & & & & & & & & & & \\
\hline V7 & 0.38 & 0.31 & 0.13 & 0.24 & 0.21 & 0.35 & 1.00 & & & & & & & & & & & \\
\hline V8 & 0.35 & 0.08 & 0.16 & 0.22 & 0.17 & 0.28 & 0.67 & 1.00 & & & & & & & & & & \\
\hline V9 & 0.15 & 0.09 & 0.26 & 0.28 & 0.19 & 0.24 & 0.25 & 0.28 & 1.00 & & & & & & & & & \\
\hline V10 & 0.40 & 0.25 & 0.06 & 0.32 & 0.31 & 0.52 & 0.43 & 0.33 & 0.24 & 1.00 & & & & & & & & \\
\hline V11 & 0.24 & 0.23 & 0.07 & 0.29 & 0.33 & 0.40 & 0.41 & 0.39 & 0.36 & 0.38 & 1.00 & & & & & & & \\
\hline V12 & 0.35 & 0.25 & 0.09 & 0.29 & 0.21 & 0.28 & 0.43 & 0.29 & 0.21 & 0.41 & 0.35 & 1.00 & & & & & & \\
\hline V13 & 0.37 & 0.33 & 0.14 & 0.38 & 0.30 & 0.28 & 0.46 & 0.26 & 0.11 & 0.48 & 0.35 & 0.57 & 1.00 & & & & & \\
\hline V14 & 0.35 & 0.27 & 0.05 & 0.28 & 0.17 & 0.25 & 0.40 & 0.24 & 0.08 & 0.52 & 0.33 & 0.63 & 0.57 & 1.00 & & & & \\
\hline V15 & 0.27 & 0.21 & $\overline{-}$ & 0.12 & 0.07 & 0.06 & 0.42 & 0.28 & 0.08 & 0.44 & 0.22 & 0.40 & 0.55 & 0.44 & 1.00 & & & \\
\hline V16 & 0.24 & 0.35 & 0.01 & 0.26 & 0.13 & 0.30 & 0.50 & 0.33 & 0.14 & 0.41 & 0.27 & 0.48 & 0.52 & 0.58 & 0.54 & 1.00 & & \\
\hline V17 & 0.32 & 0.29 & 0.10 & 0.12 & 0.13 & 0.18 & 0.62 & 0.44 & 0.14 & 0.41 & 0.29 & 0.47 & 0.55 & 0.49 & 0.63 & 0.68 & 1.00 & \\
\hline V18 & 0.31 & 0.06 & 0.06 & 0.13 & 0.13 & 0.13 & 0.44 & 0.77 & 0.23 & 0.17 & 0.29 & 0.23 & 0.13 & 0.18 & 0.27 & 0.26 & 0.37 & 1 \\
\hline
\end{tabular}

Fuente: Elaboración propia a partir de datos de la EOIC19 (Instituto Nacional de Estadística e Informática, 2020) 
Tabla 5

Pruebas estadísticas para elegir el análisis factorial

\begin{tabular}{ll}
\hline Pruebas Estadísticas & Valores \\
\hline & Chi-square $=2967,080$ \\
Test de esfericidad de Bartlett & Degrees of freedom $=153$ \\
& p-value $=0,000$ \\
Medida de adecuación muestral Kaiser-Meyer-Olkin & KMO $=0,842$ \\
\hline
\end{tabular}

Fuente: Elaboración propia a partir de datos de la EOIC19 (Instituto Nacional de Estadística e Informática, 2020)

Tabla 6

Elección del número de factores

\begin{tabular}{lcccc}
\hline Factor & Autovalor & Diferencia & Proporción & Acumulado \\
\hline Factor1 & 5,96116 & 4,10593 & 0,5961 & 0,5961 \\
Factor2 & 1,85523 & 0,54513 & 0,1855 & 0,7816 \\
Factor3 & 1,31009 & 0,69738 & 0,131 & 0,9126 \\
Factor4 & 0,61271 & 0,2842 & 0,0613 & 0,9739 \\
Factor5 & 0,32851 & 0,0666 & 0,0328 & 1,0067 \\
Factor6 & 0,26192 & 0,04163 & 0,0262 & 1,0329 \\
Factor7 & 0,22028 & 0,02752 & 0,022 & 1,0549 \\
Factor8 & 0,19277 & 0,07666 & 0,0193 & 1,0742 \\
Factor9 & 0,1161 & 0,00881 & 0,0116 & 1,0858 \\
Factor10 & 0,10729 & 0,06659 & 0,0107 & 1,0965 \\
Factor11 & 0,04071 & 0,05033 & 0,0041 & 1,1006 \\
Factor12 & $-0,00962$ & 0,06519 & $-0,001$ & 1,0997 \\
Factor13 & $-0,07481$ & 0,02178 & $-0,0075$ & 1,0922 \\
Factor14 & $-0,0966$ & 0,04378 & $-0,0097$ & 1,0825 \\
Factor15 & $-0,14037$ & 0,01929 & $-0,014$ & 1,0685 \\
Factor16 & $-0,15966$ & 0,08434 & $-0,016$ & 1,0525 \\
Factor17 & $-0,244$ & 0,03713 & $-0,0244$ & 1,0281 \\
Factor18 & $-0,28113$ & & $-0,0281$ & 1 \\
\hline
\end{tabular}

Fuente: Elaboración propia a partir de datos de la EOIC19 (Instituto Nacional de Estadística e Informática, 2020)

la rotación para hallar las cargas factoriales que serán analizadas. En la Tabla 7, se observa la asociación existente entre cada variable y los factores encontrados que se realiza debido a su mayor carga. Es necesario resaltar que los factores buscan agrupar las variables en torno a sus comunalidades y, al mismo tiempo, que la distancia entre factores sea mayor. En particular se puede ver que, mientras el Factor 1 se asocia a 8 variables, el Factor 2 se relaciona con 6 variables y el Factor 3 con 4 . Asimismo, es necesario precisar que la naturaleza de cada factor es distinta, pues el Factor 1 está más asociado a problemas de liquidez, el Factor 2 lo está más a los problemas de producción y el Factor 3 a problemas de operatividad.
Finalmente, se predijo el valor de cada factor para cada una de las empresas en la muestra y se procedió a estimar mediante el MCO el efecto que tuvieron los factores hallados sobre el índice de ventas de las empresas de Lima Metropolitana. En la Tabla 8, se muestran los resultados de la estimación.

De esta manera, de acuerdo con el estadístico F, se corroboró que la incorporación de las variables en el modelo es adecuada y, además, este modelo tiene una bondad de ajuste de 0,15 , lo cual resulta apropiado para un modelo de estas características. Asimismo, se encontró que solo la liquidez y la operatividad tienen efectos estadísticamente significativos al $5 \%$. La producción muestra un efecto 
Tabla 7

Cargas factoriales rotadas

\begin{tabular}{lcccc}
\hline Variables & Factor $\mathbf{1}$ & Factor 2 & Factor 3 & Unicidad \\
\hline Disminución de la demanda de sus productos o servicios & 0,3622 & 0,169 & 0,3276 & 0,733 \\
Paralización de la producción a causa de la pandemia & 0,3397 & 0,4307 & $-0,0158$ & 0,6988 \\
Dificultad en la exportación de sus productos & $-0,0689$ & 0,6145 & 0,0804 & 0,6111 \\
Desabastecimiento de materias primas e insumos del mercado nacional & 0,1928 & 0,7706 & 0,0632 & 0,3649 \\
Desabastecimiento de materias primas e insumos del extranjero & 0,0783 & 0,7758 & 0,08 & 0,3855 \\
Disminución de la inversión en maquinaria y equipos & 0,2248 & 0,6251 & 0,204 & 0,5171 \\
Retraso en el pago de facturas & 0,5035 & 0,1753 & 0,5692 & 0,3918 \\
Retraso en el cobro de facturas & 0,1988 & 0,1205 & 0,8713 & 0,1867 \\
Enfermedad de trabajadores por el coVID-19 & 0,0494 & 0,3117 & 0,32 & 0,798 \\
Pérdida de capital de trabajo & 0,5571 & 0,3237 & 0,2049 & 0,5429 \\
Altos costos para implementar planes de seguridad (personal y/o clientes) & 0,2973 & 0,3339 & 0,3531 & 0,6754 \\
Difícil accesibilidad a créditos de sus proveedores & 0,6358 & 0,2036 & 0,1713 & 0,525 \\
Falta de liquidez para la compra de insumos o materias primas & 0,722 & 0,267 & 0,0801 & 0,4011 \\
Limitaciones para acceder a fuentes de financiamiento & 0,7345 & 0,1566 & 0,0822 \\
Falta de liquidez para pago de remuneraciones de personal & 0,6859 & $-0,0618$ & 0,1893 & 0,4293 \\
Dificultad para pagar préstamos del sistema financiero & 0,7384 & 0,08 & 0,1943 & 0,4106 \\
Falta de liquidez para pagar a proveedores & 0,7208 & 0,0034 & 0,3568 & 0,3531 \\
Dificultad para cobrar a sus clientes & 0,1182 & 0,0089 & 0,7917 & 0,3591 \\
\hline
\end{tabular}

Fuente: Elaboración propia a partir de datos de la EOIC19 (Instituto Nacional de Estadística e Informática, 2020)

Tabla 8

\begin{tabular}{lcc} 
Estimación MCO & \\
\hline Variables & Coeficientes & P-Valor \\
\hline Liquidez & $-34,65$ & 0,00 \\
Producción & $-5,78$ & 0,08 \\
Operatividad & $-7,07$ & 0,01 \\
Constante & 67,84 & 0,00 \\
\hline Estadísticos & & \\
\hline F & 50,53 & \\
Prob > F & 0,00 & \\
$\mathrm{R}^{2}$ & 0,1563 & \\
Obs. & 714 & \\
\hline
\end{tabular}

Fuente: Elaboración propia a partir de datos de la EOIC19 (Instituto Nacional de Estadística e Informática, 2020)

estadísticamente significativo, pero solo al $10 \%$. En todos los casos, se observa que la relación de los factores con el índice de ventas es negativa, lo que muestra que los problemas de liquidez, producción y operatividad incidieron negativamente sobre el índice de ventas de las empresas en Lima Metropolitana durante la pandemia del COVID-19. Además, los problemas de liquidez afectaron en mayor magnitud el desempeño de las empresas, medido a través del índice de ventas.

\section{DISCUSIÓN}

Los resultados muestran que los distintos problemas enfrentados por las empresas ubicadas en Lima Metropolitana se refieren a las dificultades que tuvieron para producir, cobrar, conseguir financiamiento y cumplir con las normas establecidas por el Gobierno durante la pandemia, entre otras. Estos se pueden agrupar en tres grandes grupos de problemas o factores: liquidez, producción y 
operatividad. Además, tal como se esperaba, cada uno de estos factores afectó negativamente el desempeño empresarial, pues han sido las mayores limitaciones que tuvieron las empresas para llevar a cabo el normal desempeño de sus actividades. Ello va en línea con lo mencionado por Regente (2020), quien señala que el acceso al crédito, la capacidad productiva, la escala de producción y el nivel educativo del microempresario afectan positiva y estadísticamente el nivel de ventas de las empresas. En tal sentido, contar con problemas o limitaciones sobre alguna de estas variables llevará a efectos negativos sobre su nivel de ventas. Lo antes descrito también guarda relación con la función de producción, pues cada uno de estos factores resultan insumos de la producción o una aproximación a la tecnología o incluso la gestión que se usa en la operación de la empresa, a través de la capacidad del empresario.

Por otro lado, a pesar de que se halló que los factores más importantes que afectaron a las distintas empresas en Lima Metropolitana están asociados a los problemas de liquidez, producción y operatividad, esto no significa que hayan mostrado la misma magnitud en sus efectos sobre el índice de ventas de la empresa. Así, el factor más importante que afectó el desempeño de las empresas en Lima Metropolitana fueron los problemas asociados con la liquidez, seguido de la operatividad. Ello guarda relación con lo que señala el Ministerio de la Producción (2021), en donde se observa que solo 5,5\% de las micro, pequeñas y medianas empresas formales en el Perú accedieron a un crédito, mostrando así las limitaciones que tienen incluso las empresas formales para acceder a un crédito en el sistema financiero. Además, estos resultados también se condicen con lo hallado por Regente (2020), quien indica que uno de los factores más importantes y estadísticamente significativos fue el acceso al crédito por parte de la empresa. Además, concuerda con lo que indican Brealey et al. (2010), con relación a la importancia del uso de diversos mecanismos e instrumentos financieros que permitan a las empresas a acceder a activos reales como maquinarias, instalaciones y oficinas, o el valor de la marca y la especialización de trabajadores, etc.

En este sentido, los problemas de liquidez que tenga una empresa dan origen a otras limitaciones para acceder a capital de trabajo y activos, afectando el normal desarrollo de sus actividades. A su vez, estos problemas se ven incrementados en situaciones de crisis -como la ocurrida a causa de la pandemia del COVID-19- en donde las empresas del sistema financiero tienden a contraer el crédito con el propósito de disminuir los riesgos asociados al impago de los clientes, limitando así las opciones de financiamiento y agravando los problemas de liquidez de las empresas. Por ende, se guarda relación con lo hallado por Periche-Delgado et al. (2020), con relación al incremento de la morosidad durante la pandemia del COVID-19, y con la evidencia de Quilia (2020) sobre los desafíos que enfrentaron las mype durante la pandemia del COVID-19 en el Perú, resaltando así la importancia del financiamiento y los problemas de liquidez que enfrentaron las empresas debido a la paralización de actividades que trajeron consigo el incumplimiento en el pago de deudas a proveedores, afectando así a todo el tejido empresarial.

Finalmente, tal como encuentran Franco et al. (2019) para Ecuador, las empresas más pequeñas en el Perú tampoco acceden en gran medida al financiamiento a través del sistema financiero, incluso en el periodo prepandemia, por lo que el incremento en los problemas de liquidez de las empresas incrementa su nivel de vulnerabilidad. Ello es complementado por lo hallado por Achim et al. (2021) para las empresas en Rumania, quienes destacan también la importancia del financiamiento y la liquidez en el resultado obtenido por las empresas que sufrieron los efectos de la pandemia del COVID-19.

Por lo tanto, estos resultados se corroboran con la evidencia nacional e internacional acerca de los principales factores que afectaron a las empresas durante la pandemia del COVID-19. Así, se observa que los problemas de liquidez constituyeron un factor generalizado que afectó el desempeño empresarial durante este periodo.

Los resultados antes mencionados llevan a formular otras preguntas de investigación con relación a los factores determinantes en el desempeño empresarial, tales como: ¿ $\mathrm{La}$ liquidez fue un problema determinante también para las grandes empresas?; ¿ existe un efecto diferenciado por tamaño de empresas? y ¿hay un efecto diferenciado por sector económico, teniendo en cuenta que las restricciones a las empresas fueron diferenciadas?

\section{REFERENCIAS}

Achim, M., Safta, J., Văidean, V., Mureșan, G., \& Borlea, N. (2021). The Impact of COVID-19 on Financial Management: Evidence from Romania. Economic Research-Ekonomska Istraživanja, 1-26. https://doi.org/10.1080/1331677X.2021.1922090

Brealey, R., Myers, S., \& Allen, F. (2010). Principios de finanzas corporativas. México D.F.: Editorial McGraw-Hill Interamericana Editores S.A.

Cameron, C., \& Trivedi, P. (2009). Microeconometrics Using Stata. Texas: Stata Press Publication.

Coase, R. (1994). La empresa, el mercado y la ley. Madrid: Alianza Editorial. 
Franco, M., Gómez, C., \& Serrano, K. (2019). Determinantes del acceso al crédito para la Pyme del Ecuador. Revista Conrado, 15(67), 295-303. Recuperado de https://conrado.ucf.edu.cu/index.php/conrado/article/ view/961

Instituto Nacional de Estadística e Informática. (2020). Encuesta de opinión sobre el impacto del COVID-19 en las empresas. Recuperado de http://m.inei.gob.pe/ media/MenuRecursivo/boletines/resultados-encuesta-covid-lima metropolitana-2020.pdf

Instituto Peruano de Economía. (2021). Boletín de discusión. Impacto de la pandemia sobre el tejido empresarial formal. Recuperado de https://www.ipe.org.pe/ portal/wp-content/uploads/2021/09/Boletin-impacto-de-la-pandemia-sobre-las-empresas-formales.pdf

Ministerio de la Producción. (2021). Las MIPYME en cifras 2019. Recuperado de https://ogeiee.produce.gob. pe/index.php/en/shortcode/oee-documentos-publicaciones/publicaciones-anuales/item/972-las-mipyme-en-cifras-2019

Mochón, F. (2009). Economía, teoría y política (6. ${ }^{a}$ ed.). Madrid: Editorial McGraw-Hill.
Periche-Delgado, G., Ramos-Farroñan, E., \& Chamolí-Falcón, A. (2020). La morosidad ante un confinamiento del Covid-19 en la Caja Rural de Ahorro y Crédito Raíz, Perú. Investigación Valdizana, 14(4), 206-212. Recuperado de http://revistas.unheval.edu. pe/index.php/riv/article/view/801

Quilia, J. (2020). Desafíos en la gestión empresarial de las MYPE en tiempos de COVID-19, Perú (Tesis para optar el grado de magíster en Administración de Negocios). Recuperada de https://hdl.handle. net/20.500.12692/48291

Regente, P. (2020). Crédito y crecimiento empresarial: Caso de las empresas textiles en Arequipa (Tesis para optar el grado de magíster en Economía). Recuperada de http://hdl.handle.net/20.500.12404/17335

Ullah, S. (2021). Financing Constraints and Firm-Level Responses to the COVID-19 Pandemic: International Evidence. Research in International Business and Finance, 59, 1-15. https://doi.org/10.1016/j.ribaf.2021.101545 